\title{
Contribution of Deep Inspiration Breath-Hold Technique for Regional Nodal Irradiation Including Mammaria Interna in Mastectomized Left-Sided Breast Cancer Patients
}

\author{
(D) Meltem DAĞDELEN, (1) Şefika Arzu ERGEN, (1) Servet iPEK, (1) Ceren BARLAS, \\ (1) Songül ÇAVDAR KARAÇAM, (1) Didem ÇOLPAN ÖKSÜZ \\ Department of Radiation Oncology, İstanbul University-Cerrahpasa, Cerrahpasa Medical Faculty, Istanbul-Turkey
}

\begin{abstract}
OBJECTIVE
This study aim to investigate the feasibility and the cardiac and lung-sparing value of Deep Inspiration Breath-Hold (DIBH) technique compared to the Free Breathing (FB) technique among left-sided breast

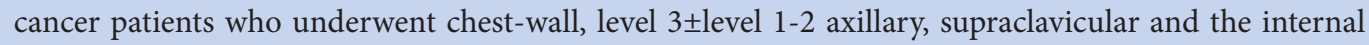
mammary nodes (IMN) irradiation.
\end{abstract}

\section{METHODS}

Ten patients who underwent the modified radical mastectomy and were treated with adjuvant radiotherapy were included in this study. All patients underwent CT simulation during FB and DIBH. Audiovisual guidance was used. Target volumes included chest-wall and regional nodes. The treatment plans and dose-volume histograms that were created on both CT scans were used to compare doses to heart, ventricle, left anterior descending artery (LAD) and lung.

\section{RESULTS}

The mean heart dose was reduced from 6,4 Gy to 3,3 Gy using DIBH technique. Heart V20, V30 and V40 and maximum dose were significantly decreased in the DIBH plans compared to FB. For LAD coronary artery, there was a significant reduction in mean dose from 42,5 Gy to 20,5 Gy in DIBH plans. There was a significant reduction in mean dose to the ipsilateral lung (ilung); V5, V10, V20 in DIBH plans.

\section{CONCLUSION}

Patients with locally advanced left-sided breast cancer require additional attention to improve heart and lung sparing to reduce late cardiovascular events and secondary cancer risks. DIBH technique led to significant reductions in heart, ventricle, $\mathrm{LAD}$, left lung DVH parameters without compromising the dose coverage to PTV in patients treated with chest-wall and lymphatic irradiation, including IMN.

Keywords: Breast cancer; chest wall irradiation; deep inspiration breath hold; free breath; lymphatic radiotherapy. Copyright $\odot$ 2020, Turkish Society for Radiation Oncology

\section{Introduction}

Breast cancer is the most common cancer and is one of the leading causes of cancer-related deaths among women. Adjuvant radiotherapy is the standard management after breast-conserving surgery, as well as after mastectomy in lymph node-positive disease. Many randomized trials have demonstrated that postopera-
Received: April 02, 2020

Accepted: April 03, 2020

Online: September 01, 2020

Accessible online at:

www.onkder.org

OPEN ACCESS This work is licensed under a Creative Commons

Attribution-NonCommercial 4.0 International License.
Dr. Meltem DAĞDELEN

Radyasyon Onkolojisi Anabilim Dalı,

İstanbul Üniversitesi-Cerrahpaşa,

Cerrahpaşa Tıp Fakültesi,

İstanbul-Turkey

E-mail: meltemdagdelen@windowslive.com 
tive radiotherapy reduces the risk of local recurrence and death rates from breast cancer and improves overall survival rate.[1] Furthermore, recent trials emphasized the importance of adding regional nodal irradiation to the whole breast or chest wall in women with node-positive or high-risk node-negative early breast cancer.[2,3] However, these benefits from radiotherapy may decrease due to the increased morbidity and mortality rates from heart disease, especially among women who received radiotherapy for left-sided breast cancer.[4-6] The increased risk of cardiac events is mainly related to the higher irradiated cardiac volumes and a considerable amount of radiation the heart. The other factors, such as the patient's baseline cardiac risk, tobacco use, and comorbidities, such as diabetes, hypertension, cardiotoxic chemotherapy and hormonotherapy regimes, may increase the effects of radiotherapy on the heart.[7-10] However, there is no detailed knowledge on the magnitude of interaction of these factors and the critical structures of the heart for radiotherapy. Furthermore, the relation between specific cardiac volumes and heart disease has not been clearly documented.[8-10] Thus, the best strategy is to keep the heart dose as low as possible. In addition, pulmonary toxicity, such as radiation pneumonitis, fibrosis and radiological abnormalities, can be seen, especially after breast with internal mammary nodes (IMN) and supraclavicular lymph nodes irradiation.[11-13] Even, some studies have noted an increased risk of ipsilateral lung cancer 10 years after the radiotherapy for smoker breast cancer patients.[10,14-16]

Advances in radiotherapy techniques, such as intensity-modulated radiotherapy (IMRT), volumetric-modulated arc therapy (VMAT), have been widely used to minimize the irradiation of normal tissues. However, a greater volume of lung and heart may receive a low dose, which translates into a relatively high level of mean heart and lung dose.[10,17-19] Another technique is the deep inspiration breath hold (DIBH) that takes advantage of a more favorable position of the heart during inspiration to minimize heart doses during the radiotherapy. Several studies performed a dosimetric comparison of Free Breathing (FB) and DIBH technique in left-sided breast cancer patients. They demonstrated that DIBH plans show reductions of dose to the heart compared to FB plans. [20-29] Chest wall radiotherapy, with inclusion of regional lymph nodes irradiation, may further enhance the dose to the heart and lung tissue.[11] However, there is a scarcity of data about the benefits of the DIBH technique in reducing heart dose in the chest wall and peripheral lym- phatic irradiation included IMN.[21,23,25-29] Also, there are conflicting results on lung sparing with DIBH technique in these groups of patients.

This study aims to investigate the feasibility and the cardiac and lung-sparing value of the DIBH technique compared to the FB technique among left-sided breast cancer patients who underwent chest wall, level $3 \pm$ level 1-2 axillary, supraclavicular and the IMN lymph nodes irradiation.

\section{Materials and Methods}

\section{Patients}

Chest-wall and regional lymph node irradiation treatment indication were administered for 18 left-sided breast cancer patients between 2014-2016. Five of the 18 patients who were treated with the DIBH technique were not included in this analysis because volumetric modulated arc therapy was used. Three of the 18 patients could not adapt to the DIBH technique and training since one patient was speaking a foreign language and the other two patients had COPD (Chronic Obstructive Pulmonary Disease). Ten of the 18 patients who were treated using the DIBH technique were evaluated in this analysis.

The mean age was 47 (range: $39-58$ ) years. Two patients were postmenopausal. None of them had a history of cardiac events, such as myocardial infarction. One patient was ex-smoker and one patient had hypertension and diabetes mellitus. Histopathological types, stage, and receptor status are demonstrated in Table 1. All patients completed four cycles of adriamycin and cyclophosphamide, followed by four cycles of docetaxel

Table 1 Tumor characteristics

Tumor characteristics

n

\begin{tabular}{ll} 
Histopathology & \\
IDC & 8 \\
Mix Type (IDC+ILC) & 2 \\
Stage & \\
II & 2 \\
III & 8 \\
ER/PR & \\
Positive & 9 \\
Negative & 1 \\
HER+2 & \\
Positive & 5 \\
Negative & 5 \\
\hline
\end{tabular}

IDC: Invasive ductal carcinoma; ILC: Invasive lobular carcinoma 
with or without trastuzumab. Three patients received neoadjuvant chemotherapy before surgery, and the others were received adjuvant chemotherapy. All patients underwent chest wall and regional nodal irradiation, including the IMN and supraclavicular nodes.

\section{Simulation}

All patients were informed about the DIBH technique, and each patient received a 15-minute training session before the planning computed tomography (GE Lightspeed 16) scan. During training, CT scanning and treatment, patients were immobilized in the supine position and their arm placed above the head. The 6-neon marker block was stabilized with tape over the xiphoid process. The Real-Time Position Management (RPM) system (version 1.7.5, Varian Medical Systems, Palo Alto, CA, USA) monitored the vertical position of an external marker block and allowed treatment delivery only when this marker was located within a predefined gating window. Audio guidance and visual feedback (video eyewear) were used to help the patient maintain a stable gating level. We controlled the breathing amplitude by visual feedback in the $2 \mathrm{~mm}$ width of the gating window, which was individually set to the mean amplitude of the stable DIBH plateau $\pm 1 \mathrm{~mm}$. For the DIBH scan, the respiration pattern of the patient was traced by the RPM system. Scanning was manually started when the breathing amplitude marker reached the gating window. The scanning time lasted approximately 20 seconds and all of the ten patients succeeded in completing the scan during one DIBH cycle. Scans were obtained with a $2.5 \mathrm{~mm}$ slice width from the mandible to the upper abdomen. For each patient, two $\mathrm{CT}$ scans were obtained; the first during FB and the second during DIBH.

The FB and DIBH CT images were transferred to the Eclipse Treatment Planning System. Target volumes and the normal tissues were separately contoured based on the Radiation Therapy Oncology Group (RTOG) contouring atlas guidelines by the same experienced physician on the FB and the DIBH CT. Clinical target volume(CTV) included chest wall, level $3 \pm$ level 1-2 axillary lymphatics, supraclavicular lymph nodes and the IMN. Planning target volumes (PTV) were generated by adding a $5 \mathrm{~mm}$ margin to the CTV, limited to the midline, and shrunk $3 \mathrm{~mm}$ from the skin as well as the chest wall lung interference. Organs at risk (OAR), such as heart, ventricles, atriums, left anterior descending artery (LAD), ipsilateral lung (ilung), contralateral lung, contralateral breast, esophagus, and thyroid, were delineated. Heart substructures and LAD were contoured according to heart atlas.[30] Margin of $0.5 \mathrm{~cm}$ was given around LAD.

\section{Treatment Planning}

Forward-planned IMRT plans were generated for each patient on FB and DIBH CT scans using the Eclipse version 8.6 treatment planning system. All plans were performed by the same medical physicist. We used a tangential chest wall and oblique supraclavicular fields with unique isocenter at the junction between the supra-clavicular field and the chest wall tangents. IMN coverage was included in tangential fields. A customized $1 \mathrm{~cm}$ thick bolus material was applied to the chest wall. Through a trial and error process, the optimized field-in field plans were determined by the evaluation of the 3D dose distribution and dose-volume histogram. The energy of the photon beams was $6 \mathrm{MV}$ for tangential fields; in some cases, to increase dose coverage in-depth, the energy of the subfields was also $15 \mathrm{MV}$. The patients received $50 \mathrm{~Gy}$ in 25 fractions for chest wall, 46 Gy in 23 fractions for a regional node.

A dose-volume histogram was generated for each technique. For each patient, target coverage and normal tissue dosimetry were analyzed on all two plans. Plans were optimized for coverage of the PTV with 93-105\% of the prescribed dose. All patients were treated with the DIBH technique with audio-visual guidance. Before the treatment, the reference respiratory curve from the RPM taken at CT simulation was imported into the Rapid arc (Varian Medical Systems, Palo Alto, CA). During the treatment inspiration level, breath-hold duration should be matched to reference. When the breathing signal falls outside this level, the treatment was stopped.

\section{Statistical Analysis}

For each patient, dose-volume histograms (DVHs) were obtained from the treatment plans performed in the two different techniques. Doses to target volumes and OARs were analyzed and the percent dose reduction in PTVs and OARs by DIBH were determined. The comparison of the doses receiving by PTV and OARs in both groups was performed using a paired t-test. The normality of all data was checked by the Shapiro-Wilk test. Wilcoxon test was used to compare non-parametric values. The computer software SPSS version 21 for Windows (IBM Corp. Armonk, NY) was used for all statistical analysis and $\mathrm{p}<0.05$ was considered statistically significant. For purposes of our study, we performed a retrospective analysis with appropriate Local Ethics Committee approval dated October 2, 2018 number of $\mathrm{A} 21$. 


\section{Results}

The comparison of treatment planning data for PTVs and organs at risk for 10 patients with FB and DIBH technique is summarized in Table 2. The DVHs demonstrated that the heart, LAD coronary artery and ipsilateral lung doses were reduced in the DIBH technique (Fig. 1).

\section{Target Volumes and Coverage}

The mean PTV volume of supraclavicular-axillary nodes and IMN were significantly increased in DIBH compared to $\mathrm{FB}$, respectively $(\mathrm{p}=0.005 ; \mathrm{p}=0.017)$. However, the coverage of PTV of the chest wall, supraclavicular-axillary nodes and IMN were comparable between the FB and DIBH plans ( $\mathrm{p}=0.49$; $\mathrm{p}=0.99$; $\mathrm{p}=0.13)$. The dose distribution and medial-lateral tangential fields for both plans were similar.

\section{Organs at Risk (OAR) \\ Cardiac Doses}

During deep inspiration, the lung volumes in the treatment field were increased due to the diaphragm motion and the heart moved away from the chest wall so separated from the high dose region. The mean heart dose was significantly reduced from 6,4Gy (range 6.4/+1.5 Gy) to $3.3 \mathrm{~Gy}$ (range 3.3-/+0.7 Gy) with $\mathrm{DIBH}$ technique compared to FB. Although in nine of ten patients, the mean heart dose was above $5 \mathrm{~Gy}$ in the FB plan, all of these patients were able to meet the mean

Table 2 Comparison of the dosimetric parameters for PTVs and organ at risk with FB and DIBH

\begin{tabular}{|c|c|c|c|}
\hline & FB & DIBH & $\mathbf{p}$ \\
\hline \multicolumn{4}{|l|}{ Chest wall } \\
\hline Volume & $577.06+/-114 \mathrm{cc}$ & $625.08+/-147 \mathrm{cc}$ & 0.08 \\
\hline V46 Gy (\%) & $97 \%+/-1.2$ & $97 \%+/-0.5$ & 0.49 \\
\hline D max (chest wall) & $54+/-0.5 G y$ & $55.3+/-0.5 \mathrm{~Gy}$ & 0.11 \\
\hline \multicolumn{4}{|l|}{ MI } \\
\hline Volume & $2+/-0.8 c c$ & $4.5+/-2.7 c c$ & 0.005 \\
\hline V40Gy (\%) & $99.6 \%+/-1$ & $99.7 \%+/-0.5$ & 0.99 \\
\hline \multicolumn{4}{|l|}{ Supraclavicular+level 3 lymph nodes } \\
\hline Volume & $39+/-12 c c$ & $50+/-16 c c$ & 0.017 \\
\hline V43 Gy (\%) & $99.6 \%+/-0.7$ & $99.8 \%+/-0.4$ & 0.13 \\
\hline \multicolumn{4}{|l|}{ Heart } \\
\hline Mean dose (Gy) & $6.4+/-1.5$ & $3.3+/-0.7$ & $<0.001$ \\
\hline D max (Gy) & $49.8+/-1.7$ & $47.3+/-2.0$ & 0.01 \\
\hline V20 Gy (\%) & $11.08+/-3.7$ & $4.6+/-1.8$ & 0.002 \\
\hline V30 Gy (\%) & $8.9+/-3.2$ & $2.4+/-1.1$ & $<0.001$ \\
\hline V40 Gy (\%) & $6.9+/-2.5$ & $1.6+/-1.25$ & 0.001 \\
\hline \multicolumn{4}{|l|}{ LAD coronary artery } \\
\hline Mean dose (Gy) & $42.6+/-4.9$ & $20.5+/-8.1$ & $<0.001$ \\
\hline Max dose (Gy) & $48.8+/-1.8$ & $34.1+/-9.5$ & 0.001 \\
\hline \multicolumn{4}{|l|}{ Left ventricle } \\
\hline Mean dose (Gy) & $10.9+/-2.7$ & $4.9+/-1.7$ & $<0.001$ \\
\hline Max dose (Gy) & $49.1+/-1.7$ & $45.1+/-2.9$ & 0.003 \\
\hline \multicolumn{4}{|l|}{ Ipsilateral lung } \\
\hline Volume (cc) & $1222.2+/-6.6$ & $1868.3+/-424.4$ & 0.001 \\
\hline Mean dose (Gy) & $17.9+/-2.7$ & $15.6+/-2.3$ & 0.002 \\
\hline V5 Gy (\%) & $53.8+/-5.8$ & $50.2+/-4.02$ & 0.008 \\
\hline V10 Gy (\%) & $42.3+/-6.7$ & $37.9+/-5.1$ & 0.007 \\
\hline V20 Gy (\%) & $35.8+/-6.2$ & $30.8+/-5.3$ & 0.002 \\
\hline \multicolumn{4}{|l|}{ Lung volume in CTV } \\
\hline D\%95 (cc) & $141.8+/-99.7$ & $116.5+/-104$ & 0.039 \\
\hline \multicolumn{4}{|l|}{ Lung volume in CTV } \\
\hline D95\% (cc)/Ipsilateral lung volume (\%) & $11.0+/-6.6$ & $6.0+/-5.4$ & 0.001 \\
\hline
\end{tabular}

PTV: Planning target volume; MI: Mammaria interna; LAD: Left anterior descending artery; CTV: Clinical target volume. Data were shown as mean values with one standard deviation 


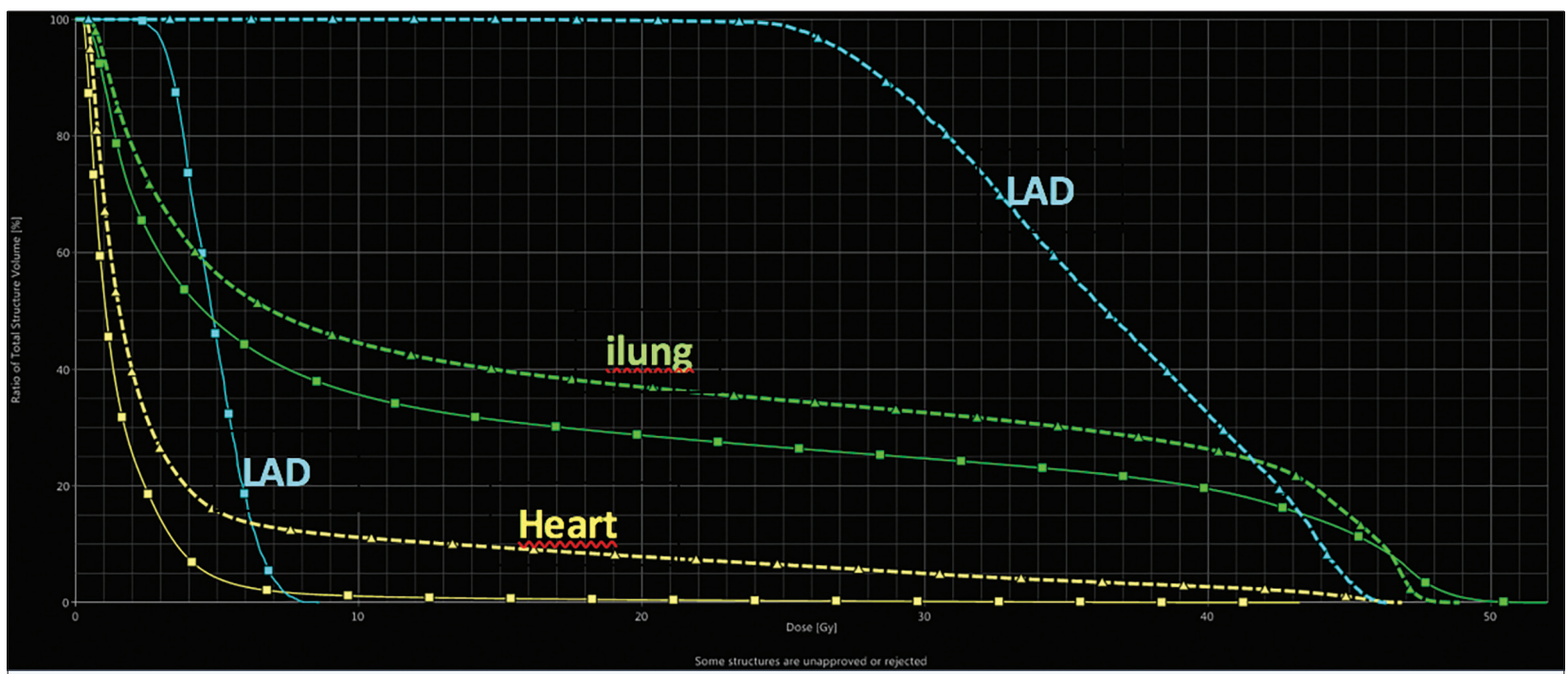

Fig. 1. Example comparison of the DVHs with DIBH (solid lines) to FB (dashed lines).

heart dose below 4 Gy in the DIBH plan. V20, V30 and V40 for the heart were significantly decreased in the DIBH plans compared to $\mathrm{FB}$, respectively $(\mathrm{p}=0.002$; $\mathrm{p}=0.001 ; \mathrm{p}=0,001)$. And the maximum dose of heart was reduced significantly in DIBH plans $(\mathrm{p}=0.01)$.

For LAD coronary artery, there was a significant reduction in mean dose from 42.5 Gy (range 42.6-/+4.9 Gy) to 20.5 Gy (range 20.5-/+8.1 Gy) in DIBH plans $(\mathrm{p}=<0.01)$. Dmax for LAD was reduced from 48.8 Gy (range $48.8-/+1.8$ gy) with FB to 34.1 Gy (range $34.1-/+9.5$ Gy) with DIBH ( $\mathrm{p}=0.001)$. Also, there was a reduction in mean and maximum doses of the left ventricle in DIBH plans compared with the FB plans, respectively $(\mathrm{p}=<0.01 ; \mathrm{p}=0.003)$.

\section{Lung Doses}

For ipsilateral lung (ilung), there was a significant reduction in V5, V10 in DIBH plans ( $\mathrm{p}=0.008$; $\mathrm{p}=0.007$ ). Mean ilung dose was decreased from 17,9 Gy with FB to 15.6 with DIBH ( $p=0.002$ ). $20 \%$ of patients in FB and $50 \%$ of patients in DIBH were able to meet ilung V20 $\leq 30 \%$. The reduction in ilung V20 with DIBH was statistically significant ( $\mathrm{p}=0.002$ ). In DIBH plans, the ipsilateral lung volumes were increased as the irradiated lung volume decreased. Irradiated lung volume/total ipsilateral volume was reduced from $11 \%$ (range11-/+6.6) to 6\% (range6-/+5.4) in DIBH plans compared to FB plans ( $\mathrm{p}=0.001)$.

We calculated the risk of radiation pneumonitis and found a reduced risk from $17 \%$ to $12 \%$.[35] The median follow-up was 34 months (range:20-50) and none of the patients developed radiation pneumonitis. Although $20 \%$ of the patients had V20 $>35 \%$, only one patient developed a ground-glass opacity (GGO) in the treatment area.

\section{Contralateral Breast Doses}

The mean and the maximum contralateral breast doses were non-significantly increased with the DIBH technique ( $\mathrm{p}=0.18$ and $\mathrm{p}=0.93$ ). The contralateral breast Dmean was 0.33 Gy with FB and 0.46 Gy with DIBH, while Dmax was 23 Gy with FB and 30 Gy with DIBH.

\section{Discussion}

Adjuvant radiotherapy is an important component of breast cancer treatment in mastectomized patients with pathologically positive lymph nodes.[1] Recent studies have supported the radiation to the chest wall and regional nodes, including IMN, even in early-stage breast cancer patients.[2,3] However, IMN irradiation doubled the mean heart dose due to anatomical position. [18] Also, the risk of pulmonary toxicity is higher, especially after chest wall with IMN and supraclavicular lymph nodes irradiation.[11] Trials have reported that the risk of death from heart disease significantly increased after 10 years in left-sided breast cancer patients compared with right-sided breast cancer patients who were treated with radiotherapy.[4-8] Darby et al. reported that the risk of cardiac diseases and cardiac mortality increase by $4-7 \%$ and $3 \%$, respectively, per $1 \mathrm{~Gy}$ in mean heart dose. $[7,8]$ The risk of radiation-related cardiac disease is increased with higher mean cardiac 
doses and there is no determined threshold dose below which there is no risk.[8-10] Also, the exact quantification of the excess risk of cardiac deaths from radiotherapy is difficult since multiple factors also have a role in cardiovascular events. [7-10] The more effective chemotherapy regimens have been used recently and the long term effects of these combinations on the heart and lung are unknown. Thus, reducing the dose to normal tissues and associated toxicity from radiotherapy may become more important considering the long-expected survival of the majority of these breast cancer patients.

Radiotherapy techniques have changed dramatically in the last two decades. Advanced radiotherapy techniques, such as IMRT and VMAT, have been favored to reduce cardiopulmonary doses as improves dose homogeneity. However, a larger volume of normal tissues may receive lower doses, and IMRT alone was not very effective in reducing heart dose.[10,17-19] Prone or lateral decubitus positioning also reduces lung and heart dose. However, these positions are not feasible in nodal radiotherapy. DIBH technique is another method that has been used to minimize irradiation of heart and lung without compromising target coverage. Several studies have demonstrated that the heart dose has been reduced with the DIBH technique compared to FB.[31] However, few studies have evaluated the role of the DIBH technique in lymphatic irradiation, including IMN. $[19,22,27,32]$ Also, most of these studies were heterogeneous and included patients with breast-conserving surgery. We prefer to evaluate the homogenous group of patients with high risk who underwent mastectomy since the prescription isodose line is closer to the heart than in patients with intact breast. Yeung et al. found that percent reduction of heart and LAD doses with DIBH were significantly larger in patients with regional nodal irradiation compared with without nodal irradiation.[27] Our study confirms reductions in all heart DVH parameters comparable to the literature.

LAD is a significant target to avoid in the pathogenesis of long-term cardiac complications. [24] Some studies revealed that LAD doses are related to coronary artery stenosis in breast cancer.[33,34] Since very few studies dealt with LAD doses, no standard protocols for the tolerance doses of LAD and there are heterogeneities about mean LAD doses in the literature. The range of mean LAD doses varied between 0,8 and 22.4 Gy with DIBH technique when the only breast was in the target volume.[35] However, the range of mean LAD doses increased to 4,1 and 23.7 Gy with DIBH technique when IMN was included in target volume. [35] Furthermore, some studies suggested that the mean dose was not representative of LAD dose since LAD is a serial structure. Nilsson et al. reported a correlation of coronary artery stenosis with hot spot areas on LAD.[34] We observed that there was $51.8 \%$ and $30 \%$ of a significant reduction in mean and Dmax LAD doses, respectively, with DIBH technique compared to FB. Dmax in our study was similar to literature, while the mean LAD dose was slightly higher than some studies.[35] It might be due to the stricter constraints on PTV coverage and PRV with a margin of $0.5 \mathrm{~cm}$ was given as the LAD displacement at DIBH was variable.[36] Also, the interobserver variability and not using guideline in the delineation of the heart and LAD may cause a large variation in the reported LAD doses between studies. We did not use intravenous contrast medium for evaluation of hearts' substructures. However, to solve this problem, only one experienced radiation oncologist contoured the volumes, and all (sub) structures of the heart were delineated according to the cardiac contouring atlas in detailed.[30]

Breast radiotherapy may also cause pulmonary complications, and radiation pneumonitis is one of the important clinical toxicities. The risk of symptomatic radiation pneumonitis is increased, especially in patients with regional nodal irradiation, including IMN even after advanced radiotherapy techniques. $[2,3,12,13]$ The most commonly shown dosimetric factors to predict $\geq$ grade II radiation pneumonitis are the percentage of ipsilateral lung volume receiving V5, V10, V20 and MLD.[37] In the DIBH technique, the total lung volume increased as the relative irradiated lung volume decreased. We observed significant dose reductions with DIBH in Dmean, V5, V10, V20 left lung. However, in some studies, no lung benefit was demonstrated with DIBH while they showed cardiac sparing.[22,26,27,31] Also, MLD, V5, V10 of ilung have been evaluated in very few studies in which supraclavicular and IMN lymphatic irradiated mastectomized patients included.[17,22,27,38] Furthermore, we showed that the predicted risk of radiation pneumonitis reduced from $17 \%$ to $12 \%$ with DIBH. All of our patients were treated with DIBH technique and we detected radiation pneumonitis radiologically in only one patient without significant clinical symptoms. This patient's ilung V20 was above $30 \%$.

It has been shown that radiation-related lung cancer may develop in long-term breast cancer survivors and increased by $11 \%$ per Gy mean lung dose.[10] Aznar et al. noted that the absolute 30-year risk of radiation-related lung cancer risk is $\sim 10 \%$ for long-term continuing smoker patients treated with regional nodal irradia- 
tion, including supraclavicular fossa and IMN in which the mean whole lung dose increased beyond $9 \mathrm{~Gy}$.[16] In the present study, the mean whole lung dose was 7.8 Gy with DIBH and showed a statistically significant dose reduction compared to $\mathrm{FB}(\mathrm{p}<0.001)$. Thus, the $\mathrm{DIBH}$ technique also reduces lung radiation exposure and minimizes the risk of radiation-related side effects.

A disadvantage of the DIBH technique is that the medial part of the contralateral breast comes closer to a higher radiation dose area. The studies found that there was a non-significant increased dose of contralateral breast.[30] This non-significant increased contralateral breast dose was found in our study, as well; the mean contralateral breast dose was 0.33 Gy with FB and 0.46 Gy with DIBH. However, these mean doses were eventually much lower than the other studies in which the mean contralateral breast dose of 2.7 Gy with DIBH. [25] Osman et al. demonstrated a significant increase in the contralateral breast dose Dmean of 2.7 Gy with VMAT techniques compared to 0.7 Gy for 3D-CRT techniques.[21] Since the risk of radiation-induced secondary cancers was found to be increased for doses of more than $1 \mathrm{~Gy}$, especially in young women less than 40 years old, it is crucial to consider contralateral breast tissue during planning.[39]

Although our study was a retrospective study with a limited number of patients, a homogenous group of patients was evaluated. Chest wall and regional lymph nodes, including IMN, were irradiated in all patients. IMN volume in our study was significantly larger in DIBH compared with FB. It would depend on increasing the length of internal mammary region craniocaudally during DIBH, probably because of the expanded intercostal distance during inspiration. All of our patients were planned with a wide tangent, forward-planned IMRT technique that has been favored by studies concerning superior IMN dose coverage and a further reduction in cardiac doses compared to other techniques.[21] In our study, there was no statistically significant difference between FB and DIBH in target volume coverage parameters. However, the Dmax doses were higher compared to other studies, which was probably because of the more rigid constraints on PTV coverage.

Most of the studies have not reported the patients compliance with DIBH. Even, some studies noted that $11-60 \%$ of patients did not complete planned radiotherapy with breathhold.[38] In the present study, the compliance of our patients to the DIBH technique was high because of the patient training before the procedure. We also used the audiovisual guidance that results in high accuracy and reproducible frequency and amplitude. Also, audiovisual guidance enables us to keep the gating window narrower that reduces the intrafractional motion of the target volumes. When compared to the other studies in which $4 \mathrm{~mm}$ width of gating window has been used, we used a stricter 2 $\mathrm{mm}$ gating window of breathing amplitude with audiovisual guidance.[32] Furthermore, consecutive patient eligibility in our study leads our results more generalizability to other populations receiving left-sided breast irradiation, including IMN.

\section{Conclusion}

In conclusion, the DIBH technique compared to $\mathrm{FB}$ led to a significant reductions in all heart, ventricle, LAD, left lung DVH parameters without compromising the dose coverage to PTV in left-sided breast cancer patients treated with chest wall and lymphatic irradiation, including IMN. Although lung doses were slightly higher than some of the studies, clinical radiation pneumonitis has not been observed. Due to a prolonged latency period for radiotherapy related cardiac toxicity, it is too early to give clinical results. Recently increased use of chest wall irradiation with nodal radiotherapy included IMN and systemic treatments with known pulmonary and cardiac side effects may enhance morbidity. Thus, it is crucial to implement simple and highly effective DIBH techniques in daily clinical practice for suitable left-sided breast cancer patients since even small dose reductions given to heart, LAD, the lung may decrease late cardiovascular events and secondary cancer risks.

Peer-review: Externally peer-reviewed.

Conflict of Interest: On behalf of all authors, the corresponding author states that there is no conflict of interest.

Ethics Committee Approval: For purposes of our study, we performed a retrospective analysis with appropriate Local Ethics Committee approval dated October 2, 2018 number of A21.

Financial Support: This research received no specific grant from any funding agency in the public, commercial, or notfor-profit sectors.

Authorship contributions: Concept - M.D., Ş.A.E.; Design - S.Ç.K., D.Ç.Ö.; Supervision - S.Ç.K., D.Ç.Ö.; Materials M.D., Ş.A.E.; Data collection and/or processing - C.B., S.İ.; Data analysis and/or interpretation - Ş.A.E., C.B.; Literature search - M.D., C.B.; Writing - M.D., S..A.E., C.B.; Critical review - S.Ç.K., D.Ç.Ö. 


\section{References}

1. EBCTCG (Early Breast Cancer Trialists' Collaborative Group), McGale P, Taylor C, Correa C, Cutter D, Duane F, et al. Effect of radiotherapy after mastectomy and axillary surgery on 10-year recurrence and 20 -year breast cancer mortality: meta-analysis of individual patient data for 8135 women in 22 randomised trials. Lancet 2014;383(9935):2127-35.

2. Poortmans PM, Collette S, Kirkove C, Van Limbergen E, Budach V, Struikmans H, et al. Internal Mammary and Medial Supraclavicular Irradiation in Breast Cancer. N Engl J Med 2015;373(4):317-27.

3. Whelan TJ, Olivotto IA, Parulekar WR, Ackerman I, Chua BH, Nabid A, et al. Regional Nodal Irradiation in Early-Stage Breast Cancer. N Engl J Med 2015;373(4):307-16.

4. Favourable and unfavourable effects on long-term survival of radiotherapy for early breast cancer: an overview of the randomised trials. Early Breast Cancer Trialists' Collaborative Group. Lancet 2000;355(9217):1757-70.

5. Hooning MJ, Aleman BM, van Rosmalen AJ, Kuenen MA, Klijn JG. Cause-specific mortality in long-term survivors of breast cancer: a 25-year follow-up study. Int J Radiat Oncol Biol Phys 2006;64(4):1081-91.

6. Henson KE, McGale P, Taylor C, Darby SC. Radiation-related mortality from heart disease and lung cancer more than 20 years after radiotherapy for breast cancer. Br J Cancer 2013;108(1):179-82.

7. Hooning MJ, Botma A, Aleman BM, Baaijens $\mathrm{MH}$, Bartelink H, Klijn JG, et al. Long-term risk of cardiovascular disease in 10-year survivors of breast cancer. J Natl Cancer Inst 2007;99(5):365-75.

8. Darby SC, Ewertz M, McGale P, Bennet AM, Blom-Goldman U, Brønnum Det al. Risk of ischemic heart disease in women after radiotherapy for breast cancer. N Engl J Med 2013;368(11):987-98.

9. Chargari C, Kirov KM, Bollet MA, Magné N, Védrine L, Cremades S, et al. Cardiac toxicity in breast cancer patients: from a fractional point of view to a global assessment. Cancer Treat Rev 2011;37(4):321-30.

10. Taylor C, Correa C, Duane FK, Aznar MC, Anderson SJ, Bergh J, et al. Estimating the Risks of Breast Cancer Radiotherapy: Evidence From Modern Radiation Doses to the Lungs and Heart and From Previous Randomized Trials. J Clin Oncol 2017;35(15):1641-9.

11. Gokula K, Earnest A, Wong LC. Meta-analysis of incidence of early lung toxicity in 3-dimensional conformal irradiation of breast carcinomas. Radiat Oncol 2013;8:268.

12. Kunkler IH. Radiotherapy of the regional lymph nodes: shooting at the sheriff? Breast 2009;18 Suppl 3:S112-20.

13. Matzinger O, Heimsoth I, Poortmans P, Collette L,
Struikmans H, Van Den Bogaert W, et al. Toxicity at three years with and without irradiation of the internal mammary and medial supraclavicular lymph node chain in stage I to III breast cancer (EORTC trial 22922/10925). Acta Oncol 2010;49(1):24-34.

14. Darby SC, McGale P, Taylor CW, Peto R. Long-term mortality from heart disease and lung cancer after radiotherapy for early breast cancer: prospective cohort study of about 300,000 women in US SEER cancer registries. Lancet Oncol 2005;6(8):557-65.

15. Prochazka M, Hall P, Gagliardi G, Granath F, Nilsson $\mathrm{BN}$, Shields PG, et al. Ionizing radiation and tobacco use increases the risk of a subsequent lung carcinoma in women with breast cancer: case-only design. J Clin Oncol 2005;23(30):7467-74.

16. Aznar MC, Duane FK, Darby SC, Wang Z, Taylor CW. Exposure of the lungs in breast cancer radiotherapy: A systematic review of lung doses published 2010-2015. Radiother Oncol 2018;126(1):148-54.

17. Lohr F, El-Haddad M, Dobler B, Grau R, Wertz HJ, Kraus-Tiefenbacher $U$, et al. Potential effect of robust and simple IMRT approach for left-sided breast cancer on cardiac mortality. Int J Radiat Oncol Biol Phys 2009;74(1):73-80.

18. Taylor CW, Wang Z, Macaulay E, Jagsi R, Duane F, Darby SC. Exposure of the Heart in Breast Cancer Radiation Therapy: A Systematic Review of Heart Doses Published During 2003 to 2013. Int J Radiat Oncol Biol Phys 2015;93(4):845-53.

19. Jin GH, Chen LX, Deng XW, Liu XW, Huang Y, Huang $\mathrm{XB}$. A comparative dosimetric study for treating left-sided breast cancer for small breast size using five different radiotherapy techniques: conventional tangential field, filed-in-filed, tangential-IMRT, multibeam IMRT and VMAT. Radiat Oncol 2013;8:89.

20. Vikström J, Hjelstuen MH, Mjaaland I, Dybvik KI. Cardiac and pulmonary dose reduction for tangentially irradiated breast cancer, utilizing deep inspiration breathhold with audio-visual guidance, without compromising target coverage. Acta Oncol 2011;50(1):42-50.

21. Osman SO, Hol S, Poortmans PM, Essers M. Volumetric modulated arc therapy and breath-hold in image-guided locoregional left-sided breast irradiation. Radiother Oncol 2014;112(1):17-22.

22. Remouchamps VM, Vicini FA, Sharpe MB, Kestin LL, Martinez AA, Wong JW. Significant reductions in heart and lung doses using deep inspiration breath hold with active breathing control and intensity-modulated radiation therapy for patients treated with locoregional breast irradiation. Int J Radiat Oncol Biol Phys 2003;55(2):392-406.

23. Nguyen MH, Lavilla M, Kim JN, Fang LC. Cardiac sparing characteristics of internal mammary chain radiotherapy using deep inspiration breath hold for 
left-sided breast cancer. Radiat Oncol 2018;13(1):103.

24. McIntosh A, Shoushtari AN, Benedict SH, Read PW, Wijesooriya K. Quantifying the reproducibility of heart position during treatment and corresponding delivered heart dose in voluntary deep inhalation breath hold for left breast cancer patients treated with external beam radiotherapy. Int J Radiat Oncol Biol Phys 2011;81(4):569-76.

25. Hjelstuen MH, Mjaaland I, Vikström J, Dybvik KI. Radiation during deep inspiration allows loco-regional treatment of left breast and axillary-, supraclavicularand internal mammary lymph nodes without compromising target coverage or dose restrictions to organs at risk. Acta Oncol 2012;51(3):333-44.

26. Rochet N, Drake JI, Harrington K, Wolfgang JA, Napolitano B, Sadek BT, et al. Deep inspiration breathhold technique in left-sided breast cancer radiation therapy: Evaluating cardiac contact distance as a predictor of cardiac exposure for patient selection. Pract Radiat Oncol 2015;5(3):127-34.

27. Yeung R, Conroy L, Long K, Walrath D, Li H, Smith $\mathrm{W}$, et al. Cardiac dose reduction with deep inspiration breath hold for left-sided breast cancer radiotherapy patients with and without regional nodal irradiation. Radiat Oncol 2015;10:200.

28. Mohamad O, Shiao J, Zhao B, Roach K, Ramirez E, Vo DT, et al. Deep inspiration breathhold for left-sided breast cancer patients with unfavorable cardiac anatomy requiring internal mammary nodal irradiation. Pract Radiat Oncol 2017;7(6):361-7.

29. Stranzl H, Zurl B, Langsenlehner T, Kapp KS. Wide tangential fields including the internal mammary lymph nodes in patients with left-sided breast cancer. Influence of respiratory-controlled radiotherapy (4D-CT) on cardiac exposure. Strahlenther Onkol 2009;185(3):155-60.

30. Feng M, Moran JM, Koelling T, Chughtai A, Chan JL, Freedman L, et al. Development and validation of a heart atlas to study cardiac exposure to radiation following treatment for breast cancer. Int J Radiat Oncol Biol Phys 2011;79(1):10-8
31. Sixel KE, Aznar MC, Ung YC. Deep inspiration breath hold to reduce irradiated heart volume in breast cancer patients. Int J Radiat Oncol Biol Phys 2001;49(1):199204.

32. Pedersen AN, Korreman S, Nyström H, Specht L. Breathing adapted radiotherapy of breast cancer: reduction of cardiac and pulmonary doses using voluntary inspiration breath-hold. Radiother Oncol 2004;72(1):53-60.

33. Correa CR, Litt HI, Hwang WT, Ferrari VA, Solin LJ, Harris EE. Coronary artery findings after left-sided compared with right-sided radiation treatment for early-stage breast cancer. J Clin Oncol 2007;25(21):30317.

34. Nilsson G, Holmberg L, Garmo H, Duvernoy O, Sjögren I, Lagerqvist B, et al. Distribution of coronary artery stenosis after radiation for breast cancer. J Clin Oncol 2012;30(4):380-6.

35. Bergom C, Currey A, Desai N, Tai A, Strauss JB. Deep Inspiration Breath Hold: Techniques and Advantages for Cardiac Sparing During Breast Cancer Irradiation. Front Oncol 2018;8:87.

36. Wang X, Pan T, Pinnix C, Zhang SX, Salehpour M, Sun TL, et al. Cardiac motion during deep-inspiration breath-hold: implications for breast cancer radiotherapy. Int J Radiat Oncol Biol Phys 2012;82(2):708-14.

37. Marks LB, Bentzen SM, Deasy JO, Kong FM, Bradley JD, Vogelius IS, et al. Radiation dose-volume effects in the lung. Int J Radiat Oncol Biol Phys 2010;76(3 Sup$\mathrm{pl):S70-6.}$

38. Czeremszyńska B, Drozda S, Górzyński M, Kępka L. Selection of patients with left breast cancer for deep-inspiration breath-hold radiotherapy technique: Results of a prospective study. Rep Pract Oncol Radiother 2017;22(5):341-8.

39. Stovall M, Smith SA, Langholz BM, Boice JD Jr, Shore $\mathrm{RE}$, Andersson $\mathrm{M}$, et al. Dose to the contralateral breast from radiotherapy and risk of second primary breast cancer in the WECARE study. Int J Radiat Oncol Biol Phys 2008;72(4):1021-30. 